\title{
In memoriam Gérard Bailloud (1919-2010)
}

\section{Claude Constantin}

\section{CpenEdition}

\section{Journals}

Édition électronique

URL : http://journals.openedition.org/rao/1391

DOI : 10.4000/rao.1391

ISSN : 1775-3732

\section{Éditeur}

Presses universitaires de Rennes

\section{Édition imprimée}

Date de publication : 31 décembre 2010

Pagination : 209-210

ISBN : 978-2-7535-1383-9

ISSN : 0767-709X

\section{Référence électronique}

Claude Constantin, «In memoriam Gérard Bailloud (1919-2010) », Revue archéologique de l'Ouest [En ligne], 27 | 2010, mis en ligne le 25 février 2012, consulté le 04 décembre 2020. URL : http:// journals.openedition.org/rao/1391 ; DOI : https://doi.org/10.4000/rao.1391

Ce document a été généré automatiquement le 4 décembre 2020

Tous droits réservés 


\title{
In memoriam Gérard Bailloud (1919-2010)
}

\author{
Claude Constantin
}

1 Gérard Bailloud, un des plus éminents fondateurs des études néolithiques françaises, s'est éteint le 30 août 2010 à Carnac où il s'était installé définitivement après son départ en retraite en 1985.

2 Après des études secondaires à Paris, il s'inscrit en licence d'Histoire et au certificat d'Ethnologie de l'université de Paris, alors le seul endroit où il pouvait suivre quelques heures d'enseignement de Préhistoire. Dans un discours de président entrant à la Société préhistorique française (1970, p. 36 et suiv.), seul texte où il évoque son parcours, il parle de son goût pour l'histoire, l'ethnologie et la préhistoire africaine qui fut renforcé par ces enseignements, mais il y confie aussi qu'à l'âge de treize ans, il écrivait déjà un roman de trente-deux pages intitulé "Les mémoires d'un homme préhistorique ».

3 À la fin des années trente, dans le cadre d'une opération destinée à trouver des emplois aux chômeurs intellectuels, il obtient une affectation à la Photothèque du Musée de l'Homme où il restera quatorze années. Dans le même discours de 1970, il indique encore « Durant ce temps, je n'ai cessé d'accroître mes connaissances en Préhistoire en lisant tout ce qui concernait cette discipline dans les importantes collections d'ouvrages et de périodiques de la bibliothèque du Musée de l'Homme ». Pour qui a pu entendre, dans des conversations où en assistant à ses cours, combien, dans la bouche de Gérard Bailloud chaque mot comptait; pour qui a pu mesurer aussi l'exhaustivité des références bibliographiques de ses ouvrages les plus fondamentaux, il est évident qu'il avait «tout » lu. De même qu'il verra le matériel de tous les musées et celui que voudront bien lui montrer tous les fouilleurs qu'il a contactés.

4 En 1946, il participe à une première fouille à la grotte du Cheval à Arcy-sur-Cure (Yonne) dont il a été le premier averti de la découverte par un ami. La direction de la fouille est assurée par André Leroi-Gourhan et les gravures pariétales de la grotte sont l'objet de la première publication de Gérard Bailloud (BSPF, 1946, p. 155 et suiv.). Mais ce sont ses participations, les années suivantes, aux fouilles pratiquées à Auvernier 
(Suisse), à la grotte Saint-Romain (Côte-d'Or) et à Armeau (Yonne) qui vont décider de sa vocation de néolithicien et lui donner le goût profond de l'étude de la céramique.

On ne peut mesurer l'apport de Gérard Bailloud aux études néolithiques sans se remémorer leur état en France au début des années cinquante. Elles sont limitées à la publication de quelques découvertes que presque personne ne sait placer dans un cadre chronologique adéquat et ce sont des chercheurs étrangers qui maîtrisent le mieux telle ou telle période du Néolithique et de l'âge du Bronze, parmi lesquels BoschGimpera, Serra-Rafols, Pigott, Childe, Daniel, Sandars, Kimmig et d'autres... En 1955, Jean Arnal, qui jouera aussi un très grand rôle dans la construction des études néolithiques françaises, peut pousser un cri d'alarme («France, réveille-toi »; BSPF, 1955, p. 347 et suiv.). En 1956, Raymond Vaufrey peut écrire « Au début de la deuxième moitié du vingtième siècle, il n'y a point en France de savant qui fasse autorité en matière de Néolithique et de Préhistoire métallique par ses fouilles passées, sa connaissance des matériaux français ainsi que de la littérature scientifique des pays limitrophes » (BSPF, 1956, p. 491 et suiv.). C'est dans une note rectificative de cet article qu'il signale la publication de l'ouvrage dont nous allons parler maintenant.

6 En effet, après une première tentative de Jean Arnal pour dresser un tableau synthétique du Néolithique français (Zephyrus, 1953, p. 311...), Gérard Bailloud et Pierre Mieg de Boofzheim publient en 1955 «Les civilisations néolithiques de la France dans leur contexte européen ». La maîtrise de la littérature que possède Gérard Bailloud, rédacteur du texte, va leur permettre et de tirer profit des travaux étrangers et de resituer les groupes culturels de France par rapport aux cultures néolithiques des pays voisins, à chaque fois qui ceci a un sens.

7 L'approche de Gérard Bailloud est résolument culturelle et, pour chaque groupe, il examine systématiquement les données concernant l'habitat, les industries lithique et osseuse, la parure, les sépultures et particulièrement la céramique dont il souligne le rôle de "technique témoin " pour toute classification culturelle. Apparaissent déjà dans cet ouvrage les principales entités culturelles du Néolithique français, qu'elles soient propres à notre sol ou rattachées aux cultures des pays voisins. Des ensembles régionaux qui ne portent pas encore de nom sont décrits et une hypothèse est proposée pour leur place chronologique. Concernant le matériel lithique, Gérard Bailloud propose déjà de placer plus tardivement que les premières manifestations du Néolithique les industries campigniennes de Louis-René Nougier dont l'ouvrage, paru en 1950, fait encore autorité alors.

En 1960 paraît l'ouvrage de Jean Arnal, Gérard Bailloud et Raymond Riquet, « Les styles céramiques du Néolithique français» qui, en plus de 200 pages, rappelle avec force l'intérêt de la céramique, livre une illustration abondante et précieuse tout en proposant déjà les éléments d'un système et d'une terminologie pour les études de la céramique.

9 En 1964 paraît la thèse que vient de soutenir Gérard Bailloud en 1962, «Le Néolithique dans le Bassin parisien ", qui sera remise à jour en 1972. Cet ouvrage fondateur des études néolithiques dans la moitié nord de la France reste aujourd'hui fondamental. Y sont définis le Groupe de Cerny, le type de Menneville apparenté au Roessen, le Groupe d'Augy-Sainte-Pallaye, toutes entités culturelles dont les découvertes ultérieures devaient confirmer la réalité. À cette construction, le discernement synthétique de l'auteur lui permettra encore d'adjoindre les groupes de Chambon et de Marcilly (1971) et, en Bretagne, le style de Groh-Collé (1975). 

lesquelles la grotte d'Artenac (Charente), l'hypogée des Mournouards (Marne), la stratigraphie de Basi (Corse), le village de Conquette (Hérault), Gérard Bailloud, entré au CNRS au début des années cinquante et mu par son goût pour la Préhistoire africaine, accomplit deux missions au Tchad et en Éthiopie où il étudie les gravures rupestres dont il prospecte les sites; au Maroc il participe aux fouilles de la nécropole néolithique d'El Kiffen.

11 Nous évoquerons ici pour les lecteurs de la Revue archéologique de l'Ouest sa contribution à l'étude du Néolithique de la façade atlantique de notre pays.

- En 1955, dans l'ouvrage cosigné avec Mieg de Boofzheim déjà évoqué, il dresse un premier tableau des groupes breton et de Charente-Maritime - Gironde du mégalithisme, insistant sur sa longue durée en Bretagne et sur la variété de ses formes.

- En 1961, (actes du premier colloque atlantique, Brest, 1963, p. 22) il propose l'existence de liens entre le Groupe de Cerny et le matériel des tertres morbihannais et du site Pinacle à Jersey.

- En 1962, est créée la civilisation d'Artenac à la suite des fouilles, avec Claude Burnez, de la grotte éponyme (Bailloud et Burnez, BSPF, p. 33 et suiv.).

- En 1975, il dresse un tableau des céramiques cannelées du Morbihan (BSPF, 1975, p. 343...) en publiant des documents inédits, en en précisant la chronologie et en ajoutant aux styles du Castellic et de Conguel définis par Jean L'Helgouach, celui de Groh-Collé.

Gérard Bailloud était très attaché à la Bretagne et à sa langue, qu'il avait apprise par ses propres moyens. Installé après sa retraite à Carnac il traduisit des textes littéraires bretons et s'intéressa à l'imprimerie en langue bretonne. Il se consacra également à la mise en valeur des archives de Zacharie Le Rouzic, le grand-père de son épouse Mauricette Jacq-Le Rouzic.

13 En plus de sa production scientifique il faut mentionner le rôle de Gérard Bailloud dans l'enseignement et la direction de la recherche mais aussi dans l'administration de cette recherche, aussi bien institutionnelle qu'associative. De 1963 à 1967 il enseigne à l'école du Louvre. De 1965 à 1968 il est directeur des Antiquités préhistoriques d'Île de France. De 1972 à 1983 il dirige, après le décès de Bohumil Soudsky, l'URA 12, équipe mixte CNRS-Université Paris I en même temps qu'il est chargé de cours à la même université.

On ne peut terminer cette évocation sans parler du rôle que Gérard Bailloud a joué à la Société préhistorique française dont il a été le secrétaire général dix-neuf années durant, de 1965 à 1983. Fin 1964, la société est sans direction et a accumulé les retards de publication; Gérard Bailloud va en diriger le redressement puis en impulser le renouveau avec la modernisation des statuts et en élever la qualité scientifique des publications. Sa détermination, son efficacité méthodique toujours discrète, son temps non compté ont permis à la SPF de poursuivre son chemin. Ce sont ces mêmes qualités qui, avec l'approfondissement permanent et exhaustif de ses connaissances, la clarté de sa pensée ont fait de celui dont nous saluons ici la mémoire un acteur majeur des études néolithiques de notre pays. 


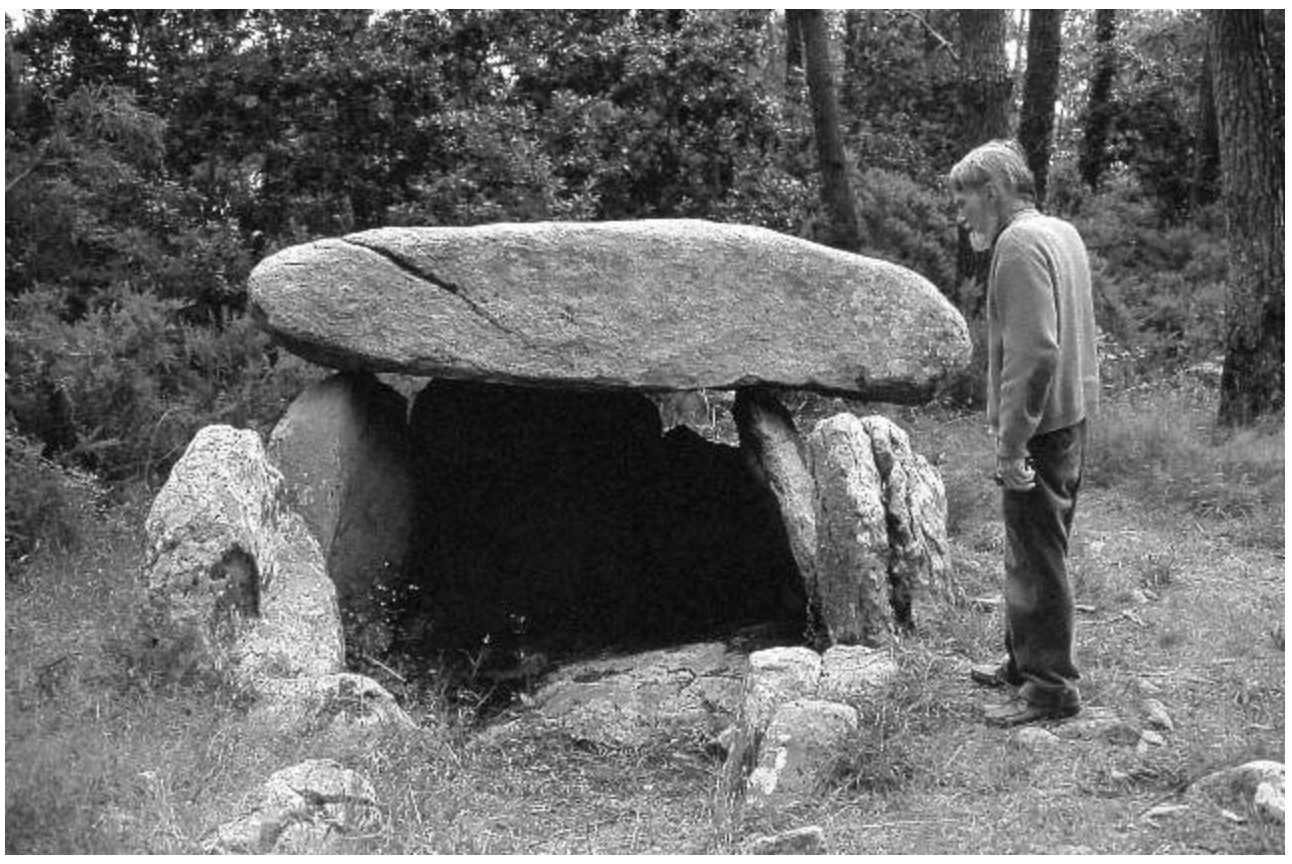

